\title{
$\angle$ Research Square \\ Rapid-SL: Identification of Higher Order Synthetic Lethal Sets with an Arbitrary Cardinality
}

\section{Mehdi Dehghan Manshadi}

Department of Chemical Engineering, School of Chemical, Petroleum and Gas Engineering, Shiraz University, Shiraz

\section{Payam Setoodeh}

Department of Chemical Engineering, School of Chemical, Petroleum and Gas Engineering, Shiraz University, Shiraz

Habil Zare ( $\nabla$ zare@uthscsa.edu )

Department of Cell Systems and Anatomy, University of Texas Health Science Center, San Antonio, San Antonio, Texas

\section{Research Article}

Keywords: Synthetic lethality, Drug target identification, Systems biology, Depth-first search, Higher order synthetic lethals, Metabolic network

Posted Date: February 16th, 2022

DOI: https://doi.org/10.21203/rs.3.rs-1350850/v1

License: (c) (1) This work is licensed under a Creative Commons Attribution 4.0 International License.

Read Full License 


\section{Abstract}

The multidrug resistance of numerous pathogenic microorganisms is a serious challenge that raises global healthcare concerns. Multi-target medications and combinatorial therapeutics are much more effective than single-target drugs due to their synergistic impact on the systematic activities of microorganisms. A synthetic lethal (SL) is a set of non-essential targets (i.e., reactions or genes) that prevent the proliferation of a microorganism when they are "knocked out" simultaneously. To facilitate the identification of SLs, we introduce Rapid-SL, a new multimodal implementation of the Fast-SL method, using the depth-first search algorithm. The advantages of Rapid-SL over Fast-SL include: (a) the enumeration of all SLs that have an arbitrary cardinality, (b) a shorter runtime due to search space reduction, (c) embarrassingly parallel computations, and (d) the targeted identification of SLs. Targeted identification is important because the enumeration of higher order SLs demands the examination of too many reaction sets. Accordingly, we present specific applications of Rapid-SL for the efficient targeted identification of SLs. In particular, we found up to $67 \%$ of all quadruple SLs by investigating about $1 \%$ of the search space. Furthermore, 307 sextuples, 476 septuples, and over 9,000 octuples are found for Escherichia coli genome-scale model, iAF1260.

\section{Introduction}

A number of human pathogenic microorganisms show multidrug resistance, which is a serious challenge in the era of global healthcare $[1,2]$. Most of these species benefit from several pathogenicity factors (i.e., the production of antigens) and broad drug-resistance mechanisms (i.e., antibiotic target mutations). Hence, disrupting the activity of only a single gene in these microorganisms does not guarantee to prevent their growth or the biosynthesis of virulence factors. Furthermore, targeting the essential reactions or genes in some pathogens may cause a significant increase in biofilm-associated reactions. This implies that single essential genes may not be proper targets for these types of microorganisms [3]. In contrast, multi-target medications and combinatorial therapeutics synergistically impress the microorganisms' systematic activities; thus, they have been recommended to be much more operative and they show less drug resistance than single targets [4].

Computational systems biology proposes powerful methodologies to address biomedical queries (e.g., human disease metabolism, the identification of potential drug targets) via a multidisciplinary systemslevel study that considers multifaceted interactions between many elements in biological networks. Constraint-based models (CBMs) are very influential in this regard. These models are successfully employed as operative mathematical representations of genome-scale metabolic models (GEMMs) by imposing the governing context- and condition-specific constraints on genome-scale metabolic network reconstructions (GENRES). CBMs can comprehensively analyze metabolic activities and examine the physiological properties of biological systems. Deploying CBMs, systematic analyses can be performed by applying the potent class of computational techniques that are available in constraint-based reconstruction and analysis (COBRA) toolbox [5]. 
Because in silico studies save significant time and expense, these methods are widely employed to identify the various effects of reaction and gene knockouts on the flux distribution of the metabolic networks of interest. These knockout studies can be implemented to identify new drug targets from three perspectives [6]: a) targeting virulence factors [3], b) metabolite-centric targeting [7-9], and c) targeting essential reactions and genes [10-14]. The last perspective is known as the most common method for identifying potential drug targets, and it is not limited to the deletion of only one reaction or gene. Synthetic lethals (SLs) are combinations of non-essential reactions or genes that are deleterious to an organism when they are disrupted simultaneously [15].

Two approaches are used to computationally identify SLs: exhaustive search and search space reduction. Exhaustive search is straightforward and has been used in some studies [14,16], but applying this approach to identify higher order SLs $(n>3)$ is not feasible due to computational time problems. Therefore, other methods are required to handle such problems by reducing the search space. Depending on the suggested criteria used to reduce the search space, some of these methods can find only a fraction of the higher order SLs, while some other methods aim to find all the SLs.

One of these methods is called "SL Finder," which is an optimization-based method for the exhaustive and targeted identification of SLs [15]. In order to reduce the search space, this method employs the fluxcoupling analysis [17] to add only one of the fully coupled reactions in the knockout list. This approach was used to discover all double and triple SLs and conduct a targeted identification of a few quadruple and quintuple SLs for iAF1260 GENRE of Escherichia coli [18].

Another method named MCSEnumerator, finds intervention strategies by enumerating the elementary modes of the dual network [19] of the corresponding metabolic network [20]. It is a powerful approach especially for metabolic engineering applications. Further improvements were made on this approach to obtain the generalized framework of MCSEnumarator and accelerate the dual calculations [21-23]. MCSEnumerator was applied to find all double to quintuple SLs in iAF1260 [20]. However, the computational time increases exponentially for SLs that have higher cardinalities, and therefore, the search procedure needs to be stopped after finding a predefined number of SLs or a time limit is reached. Alternatively, in this paper, we propose a targeted enumeration algorithm aiming to increase search efficiency.

Fast-SL is a powerful algorithm that drastically reduces the search space by purging the search space of reactions that are guaranteed not to produce SLs [24]. Fast-SL computes a flux distribution that maximizes the growth rate using a minimum value for the sum of fluxes $\left(l_{1}\right.$-norm) in order to identify flux-carrying reactions. In the next step, the algorithm searches only through these flux-carrying reactions as well as their combinations to identify SLs, which significantly reduces the search space. The authors reported the identification of 127 new synthetic lethal genes in E. coli, which had not been found by SL Finder. Also Fast-SL outperforms the MCSEnumerator by finding the same SLs about four times faster. Fast-SL provided a valuable idea for finding SLs in a reduced search space, but the implementation of this method has two major drawbacks. First, the authors developed different procedures in order to obtain 
the SLs with different cardinalities, up to quadruple SLs. Therefore, to obtain higher order SLs $(n>4)$, one must develop an entirely new procedure for each cardinality. Consequently, if one follows the implementation footsteps in the original Fast-SL, the procedure becomes extremely complicated and requires labor-intensive work to develop. The second drawback is that Fast-SL lacks an organized search method; therefore, several duplicated cases are studied in the original Fast-SL. This causes serious problems when searching for SLs with high cardinalities.

Logic transformation of model (LTM) is another method used in this field. This method changes the stoichiometry matrix (i.e., the $S$ matrix) by adding pseudo-metabolites and reactions to consider the GPRs [25]. However, the LTM method increases the size of the $S$ matrix, which in turn enlarges the problem size. Thus, more linear programming problems (LPs) must be solved to find SLs. Hence, this method becomes extremely time consuming to perform knockouts regarding higher order SLs.

As mentioned earlier, drug resistance is an important concern and identification of new drug targets based on the concept of synthetic lethality can be a suitable solution for this issue. However, comparing the effect of the different synthetic lethal sets on the metabolic network and its functionality reveals that some of the sets with higher cardinalities can make stronger and deeper effects on the network. For instance, we can divide the synthetic lethal sets into two types: a) ordinary SLs with a negligible maximum value of biomass objective function, b) "stronger" SLs for which no solution for the corresponding linear program exists. It is expected that the second type of solutions are more effective lethals and thus will provide us with targets that are harder to resist by pathogens. Based on our in silico observations, few numbers or even none of these stronger SLs can be obtained in synthetic lethal sets with two or three members while higher order SLs provide us with more of these stronger SLs.

The purpose of the current work is to develop a comprehensive and straightforward reimplementation of the Fast-SL algorithm to facilitate the identification of higher order SLs. We call our implementation Rapid-SL, which has two major steps that are iteratively performed based on the depth-first search (DFS) algorithm [26]: (1) identification of the seed space (i.e., reactions with nonzero fluxes) and (2) searching within the seed space to find the solutions. The main difference between this new implementation and the original Fast-SL is the compartmentalization of the searching process into several branches. This branching allows embarrassing parallelization [27] and prevents the examination of duplicate cases. This reduces the search space by about 35-60\% compared to Fast-SL. As in the case of Fast-SL, postprocessing of Rapid-SL results are required to make the obtained potential drug targets to druggable candidates.

In order to examine the performance of the developed method, we compared the results of Rapid-SL and Fast-SL for three microorganisms. Afterwards, we introduced three applications for Rapid-SL that could be effective for the targeted identification of higher order SLs $(n>4)$. Accordingly, we can: (1) Search among a specific list of reactions chosen consistent with a biological context (2) Apply graph-based search methods (3) Selectively enumerate the SLs among the DFS branches. Mention that the reported 
results were based on our in silico experiments. In the current work, over 9,000 octuple $(n=8)$ SL reactions were reported for E.coli using iAF1260 GEMM.

\section{Materials And Methods}

To make the identification of the higher order SLs feasible, we must reduce the search space. The knockout of a reaction set that includes only non-flux-carrying reactions does not change the flux through biomass formation reaction [24]. Therefore, to reduce the search space, we first identify and focus on the set of flux-carrying reactions, which we denote as the seed space $\left(J_{n z}\right)$ in this work. In the second step, we search for the SLs within the seed space. Moreover, each non-lethal subset of the seed space defines a new proliferating mutant strain. Using a DFS approach, we repeat the first and second steps for each of the new mutant strains (Fig. 1). This iterative process continues until certain stopping conditions are met. Each step of the process, as well as the stopping conditions, are described as follows.

\section{3-1-First Step: Identification of the seed space}

We denote the flux of reactions by $v_{j} s$. In the first step, two flux-balance-analysis (FBA)-related LPs [28] are considered and solved. These LPs lead to the identification of a flux distribution that maximizes the flux of the biomass objective function $\left(\mathrm{v}_{\text {bio }}\right)$, while the $l_{1}$-norm of the fluxes is set to its minimum value. The first LP is defined as

s.t.

$$
\min \sum_{j}\left|v_{j}\right|
$$

$$
\begin{aligned}
& v_{b i o}=v_{\text {bio, } W T} \\
& \text { S } v=0 \\
& v_{\mathrm{lb}} \leq v \leq v_{\mathrm{ub}},
\end{aligned}
$$

where $\mathrm{v}_{b i o, W T}$ is the growth of the wild-type strain calculated by solving the following LP problem:

$$
\begin{array}{ll} 
& \max v_{b i o} \\
\text { s.t. } & \\
& \mathrm{S} v=0 \\
& v_{\mathrm{lb}} \leq v \leq v_{\mathrm{ub}} .
\end{array}
$$

The goal of computing this flux distribution is to characterize the flux-carrying reactions, or the seed space.

Applying flux-variability analysis (FVA) [29] instead of computing $l_{1}$-norm of the fluxes would provide us with more information about the effect of each reaction on the biomass objective function. However, FVA is a time consuming process and using this method repetitively would cripple the whole process.

\section{3-2-Second Step: Searching within the seed space}


All combinations between the reactions in the seed space have the potential to form SLs; therefore, the exhaustive search is performed in the second step. However, when an SL is found in this step, the corresponding supersets are excluded to prevent the investigation of duplicated cases or the production of trivial answers. Furthermore, each non-lethal set identified in this step defines a proliferating mutant (i.e., a new virtual strain). This second step also includes the listing of all non-lethal sets to investigate their related proliferating mutants by removing more potential reactions in the next level of the search. Figure 2 depicts these explanations using a toy model. This step is performed in a parallel loop in the first level for the wild-type strain to decrease the wall-clock time.

\section{3-3-Backtracking and the stopping conditions}

As described by Pratapa et al. [24], removing a set of reactions that includes only non-flux-carrying reactions would have no effect on the flux of biomass formation reaction; therefore, at least one reaction in the seed space of the wild-type strain $\left(J_{n z}\right)$ should participate in each SL. Here, we generalized this statement from the wild-type strain to any virtual strain obtained during our search procedure. In other words, each reaction designated for removal in subsequent steps of the DFS algorithm should originate from the seed space of the parent virtual strain. Therefore, after we evaluate the first and the second steps for the wild-type strain, we iteratively repeat these two steps for all the resulting virtual strains identified in the second step of the previous level. Each of these mutants is treated the same as the wildtype strain; therefore, we face an iterative problem, which is handled using the DFS algorithm (the associated pseudocode is available in Supplementary Notes S2). Note that, other organized search algorithms such as breadth-first search [30] and best-first search [31] instead of DFS can be used easily in our implementation.

The search proceeds from the root node, which consists of a nonlethal set. As an example, consider a general non-lethal set, $\Delta_{m}$ with $m$ members, which is derived from the evaluation of the second step for the wild-type strain. Let $\mathrm{J}_{\mathrm{nz}}^{\Delta \mathrm{m}}$ be the seed space of the mutant strain that results from the removal of the non-lethal set of $\Delta_{\mathrm{m}}$. The set of $\mathrm{J}_{\mathrm{nz}}^{\Delta \mathrm{m}}$ is evaluated by passing the corresponding mutant to the first step. Because all the reactions in $\mathrm{J}_{\mathrm{nz}}$ and their combinations are studied in the other branches, only the fluxcarrying reactions of this mutant, which belongs to $\mathrm{J}_{\mathrm{nz}}$ are considered at this level. If there are any

reactions at this level (i.e., $\mathrm{J}_{\mathrm{nz}}^{\Delta \mathrm{m}}-\mathrm{J}_{\mathrm{nz}} \neq \varnothing$ ), the second step is triggered for all the members of $\mathrm{J}_{\mathrm{nz}}^{\Delta \mathrm{m}}-\mathrm{J}_{\mathrm{nz}}$. In Rapid-SL, backtracking occurs in three cases, and extensions cannot go deeper:

(a) when a set is found to be lethal.

(b) when no new reaction gains non-zero flux after removing a set (i.e., $\mathrm{J}_{\mathrm{nz}}^{\Delta \mathrm{m}}-\mathrm{J}_{\mathrm{nz}} \neq \varnothing$ ).

(c) when the size of the examined set reaches the maximum desired cardinality. 
Pratapa et al. [23] state that Fast-SL is not an embarrassingly parallel algorithm; however, they provide a parallel version of the Fast-SL only for the evaluation of quadruple SLs. This parallel version performs parallel calculations for some specific parts of the Fast-SL algorithm. Unlike Fast-SL, Rapid-SL is an embarrassingly parallel algorithm because it is possible to evaluate the branches of the DFS algorithm using a parallel procedure.

\section{3-4-Enumeration of synthetic lethal gene sets}

To enumerate the synthetic lethal gene sets, the same procedure is employed, except those non-zero-flux reactions obtained in each part are converted to the functioning genes using GPR rules (Supplementary Notes S3). In this work we focused on the enhancement of the identification of SL reactions, which is the main step in the process of finding SL genes. To find SL genes, other improvements can be made by involving and translating GPR rules to make further reduction in the search space prior to the main identification process. Methods such as gMCS [32] effectively use this feature for identification of synthetic lethal genes.

\section{Results}

We present our results in two parts. First, the performances of Fast-SL and Rapid-SL were compared in the identification of SLs for three microorganisms (See the supplementary Notes S4 for the comparison between Rapid-SL and duality-based methods). Then, we report the results of the three applications of Rapid-SL for the targeted enumeration of the higher order SLs. The overall computation time of the Fast$S L$ and Rapid-SL is mostly dependent on the time that is spent on the solving of the LPs. Therefore, to ensure a fair comparison between Fast-SL and Rapid-SL, we reported the number of LP problems that were solved by each approach. Furthermore, a comparison of wall-clock runtime is provided in Supplementary Notes S5. The results were obtained using a workstation with a $2.2 \mathrm{GHz}$ Intel Xeon E52696 v4 processor, which has 12 cores available for computation.

\section{4-1-Synthetic-lethals of the three microorganisms}

Table 1 shows the respective numbers of SLs with different cardinalities (up to quadruples) obtained by our implementation and obtained by the original Fast-SL. Since the SLs identified by both methods were found to be the same, the table does not report the number of these SLs found by each method.

* The exchange and diffusion reactions are not generally considered in the lethality analysis.

Table 1 indicates that our new implementation explores about $40-65 \%$ of the search space of the original Fast-SL, and it does not omit any potential cases (Supplementary files A-C). This reduction in the search space is achieved by preventing the investigation of identical cases produced in different branches.

\section{4-2-Applications of Rapid-SL}


As the maximum desired cardinality of SLs increases, there is an exponential increase in both the search space and in the number of cases to be examined in order to find all possible SLs As a result, it is not feasible to find all possible SLs with high cardinalities (e.g., octuple SLs) using the algorithms that are currently available $[20,24]$. Therefore, we take the advantage of our new implementation to effectively investigate these large search spaces. Here we introduce three applications of Rapid-SL to perform the targeted enumeration of higher order SLs.

\section{4-2-1-Searching a list of specific targets}

The simplest method to find a fraction of solutions is to specify only a limited group of reactions. However, it is not clear what reactions should be selected. These reactions may be selected from a specific subsystem or pathway that has been diagnosed as important for the growth of the microorganism. For example, we performed a search to find octuple SLs (i.e., with eight reactions in a set) among 65 core reactions introduced by Hädicke and Klamt for generating a core model from iJ01366 [34, 35]. The results are shown in Table 4 (Supplementary File D).

Our new implementation makes this analysis feasible, but at the first sight, it may seem that using Rapid$S L$ is not necessary, and it may seem sufficient to find these results using an exhaustive search because of the small number of reactions involved in the analysis. However, Rapid-SL uses a search space that is about 50 times smaller than the exhaustive search, which consequently requires an extremely timeconsuming process even for small numbers of reactions. Also, it is not feasible to perform this analysis using the original Fast-SL, because separated algorithms should be devised for the cardinality of each SL.

\section{4-2-2-Applying constraints on the branching of the DFS}

Since Rapid-SL applies the DFS algorithm to investigate the search space, it is possible to define thresholds or conditions to limit the branching and search only the more probable parts of the corresponding tree. For example, we established a criterion in which sets are allowed to branch only if their deletion reduces the growth rate of the corresponding strain by at least $1 \%$. Table 4 shows results obtained by applying this criterion to the process of identifying octuple SLs $(n=8)$ for iAF1260 (Supplementary File E). Here, the critical value of $1 \%$ was selected based on trial and error. Other values could be employed based on the studied GEMM and the growth medium. Also, other types of constraints could be defined, such as the change in the pool of a specific metabolite or the fluxes of other reactions.

\section{4-2-3-Selective enumeration among the DFS branches}

Consider the process of seeking the quadruple SLs of E.coli using iAF1260. If we group the branches of the Rapid-SL algorithm based on the number of reactions in the starting node of each branch, it is evident that the number of LPs solved in each group substantially increases as the cardinality of the starting node increases. On the other hand, the number of identified SLs per LP solved dramatically decreases 
(Table 2). Therefore, a large portion of the SLs can be identified by performing a lethality analysis on a limited number of branches.

According to Table 2, it is possible to extend only the branches in Group (I) to identify over $34 \%$ of all SLs (excluding single lethals), while about $0.65 \%$ of all LPs are examined. It should be noted that 268 SLs identified in Group (I), consist of 74 double, 98 triple, and 82 quadruple SLs. The same analyses were performed for the same microorganism ( $E$. coli) with a different genome-scale model (i.e. iJO1366) and also for a different type of microorganism (i.e. Klebsiella pneumoniae, iYL1228 [36]) to check the generalizability of this observation (Table 3 ).

It could be inferred from Table 3 that an evaluation of the branches in Group (I) is a reliable approach to find a considerable fraction of all SLs. For the GEMMs that were studied, we found up to $67 \%$ of all SLs (i.e. including double, triple and quadruple SLs) using the illustrated method by examining only about $1 \%$ of the search space that must be evaluated to find all quadruple SLs. We applied this method to find the octuple SLs of iAF1260 to investigate the efficiency of this approach for identification of higher order SLs $(n>4)$ (Table 4 and Supplementary File F).

According to Table 4, over 9,000 octuple SLs were found using the illustrated application of Rapid-SL. Based on the size of the GEMM and the maximum desired cardinality of SLs, it is possible to consider other groups of branches. For instance, Table 2 shows that evaluating both Groups (I) and (II) for iAF1260 reduces the search space by over $90 \%$ while identifying over $63 \%$ of all SLs.

\section{Discussion}

In this paper, we introduce Rapid-SL as a new implementation of Fast-SL that enables the algorithm to find higher order SLs with arbitrary cardinalities. Unlike Fast-SL, this new implementation fully supports embarrassingly parallel computations. Furthermore, compared to Fast-SL, the application of the DFS algorithm (a structured search method) decreased the number of evaluated LP problems by about 35$60 \%$. The original implementation of Fast-SL is not embarrassingly parallel and suffers from time consuming sequential computations in some of its steps. Accordingly, for larger models and higher order SLs, the difference between the computational time of Fast-SL and Rapid-SL increases and Rapid-SL becomes more and more efficient. Although Rapid-SL is not limited in terms of the cardinality of SLs, it is not feasible to seek for all SLs with higher cardinalities $(n>4)$ without using computer clusters. When using a single conventional computer, the runtime of this examination may extend to several months because of the tremendous number of potential cases. Owing to our proper implementation, Rapid-SL can effectively find a considerable portion of higher order SLs by searching only a relatively small fraction of potential cases. Accordingly, three Rapid-SL applications were introduced: a) searching among a selected list of potential reactions, b) applying constraints on the branching of the DFS, and c) selective enumeration among the DFS branches. These applications identified up to $67 \%$ of quadruple SLs by searching about $1 \%$ of the potential cases. Particularly, over 9,000 octuple synthetic reactions were found 
for iAF1260 in the third application. Accordingly, Rapid-SL can be effective for investigating large models such as genome-scale metabolic models of human cells to find drug targets with high cardinalities.

Although the first two applications find fewer SLs than the other application, they may still be useful for seeking SLs with specific biological considerations. The importance of this feature becomes clear when one considers that a single organism may have more than several thousand SLs with high cardinalities, and experimental validation of these SLs is not feasible. Therefore, both scenarios require the consideration of biological criteria when searching for useful SL sets. In future work, we will focus on defining new criteria to reduce the number of potential drug-targetable SLs.

\section{References}

[1] Jackson, R. A. \& Chen, E. S. Synthetic lethal approaches for assessing combinatorial efficacy of chemotherapeutic drugs. Pharmacology \& therapeutics162, 69-85 (2016).

[2] Van Duin, D. \& Paterson, D. L. Multidrug-resistant bacteria in the community: trends and lessons learned. Infectious Disease Clinics30, 377-390 (2016).

[3] Xu, Z., Fang, X., Wood, T. K. \& Huang, Z. J. A systems-level approach for investigating Pseudomonas aeruginosa biofilm formation. PloS one8, e57050 (2013).

[4] Silver, L. L. Multi-targeting by monotherapeutic antibacterials. Nat Rev Drug Discov6, 41-55, doi:10.1038/nrd2202 (2007).

[5] Heirendt, L. et al. Creation and analysis of biochemical constraint-based models: the COBRA Toolbox v3. 0. arXiv preprint arXiv:1710.04038 (2017).

[6] Dougherty, B. V., Moutinho Jr, T. J. \& Papin, J. Accelerating the drug development pipeline with genome-scale metabolic network reconstructions. Systems Biology6 (2017).

[7] Singh, S., Malik, B. K. \& Sharma, D. K. Choke point analysis of metabolic pathways in E. histolytica: a computational approach for drug target identification. Bioinformation2, 68 (2007).

[8] Kim, H. U., Kim, T. Y. \& Lee, S. Y. Genome-scale metabolic network analysis and drug targeting of multidrug resistant pathogen Acinetobacter baumannii AYE. Molecular BioSystems6, 339-348 (2010).

[9] Kim, H. U. et al. Integrative genome-scale metabolic analysis of Vibrio vulnificus for drug targeting and discovery. Molecular systems biology7 (2011).

[10] Chavali, A. K., Whittemore, J. D., Eddy, J. A., Williams, K. T. \& Papin, J. A. Systems analysis of metabolism in the pathogenic trypanosomatid Leishmania major. Molecular systems biology4 (2008).

[11] Thiele, I. et al. A community effort towards a knowledge-base and mathematical model of the human pathogen Salmonella Typhimurium LT2. BMC systems biology5, 1-9 (2011). 
[12] Chavali, A. K., D'Auria, K. M., Hewlett, E. L., Pearson, R. D. \& Papin, J. A. A metabolic network approach for the identification and prioritization of antimicrobial drug targets. Trends Microbio/20, 113-123, doi:10.1016/j.tim.2011.12.004 (2012).

[13] Hartman, H. B. et al. Identification of potential drug targets in Salmonella enterica sv. Typhimurium using metabolic modelling and experimental validation. Microbiology160, 1252-1266 (2014).

[14] Sigurdsson, G., Fleming, R. M., Heinken, A. \& Thiele, I. A systems biology approach to drug targets in Pseudomonas aeruginosa biofilm. PLoS One7, e34337, doi:10.1371/journal.pone.0034337 (2012).

[15] Suthers, P. F., Zomorrodi, A. \& Maranas, C. D. Genome-scale gene/reaction essentiality and synthetic lethality analysis. Mol Syst Bio/5, 301, doi:10.1038/msb.2009.56 (2009).

[16] Tymoshenko, S. et al. Metabolic needs and capabilities of Toxoplasma gondii through combined computational and experimental analysis. PLoS computational biology11, e1004261 (2015).

[17] Burgard, A. P., Nikolaev, E. V., Schilling, C. H. \& Maranas, C. D. Flux coupling analysis of genome-scale metabolic network reconstructions. Genome Res14, 301-312, doi:10.1101/gr.1926504 (2004).

[18] Feist, A. M. et al. A genome-scale metabolic reconstruction for Escherichia coli K-12 MG1655 that accounts for 1260 ORFs and thermodynamic information. Molecular systems biology3, 121 (2007).

[19] Ballerstein, K., von Kamp, A., Klamt, S. \& Haus, U.-U. Minimal cut sets in a metabolic network are elementary modes in a dual network. Bioinformatics28, 381-387 (2012).

[20] von Kamp, A. \& Klamt, S. Enumeration of smallest intervention strategies in genome-scale metabolic networks. PLoS computational biology10 (2014).

[21] Schneider, P., von Kamp, A. \& Klamt, S. An extended and generalized framework for the calculation of metabolic intervention strategies based on minimal cut sets. PLoS computational biology16, e1008110 (2020).

[22] Klamt, S., Mahadevan, R. \& von Kamp, A. Speeding up the core algorithm for the dual calculation of minimal cut sets in large metabolic networks. Bmc Bioinformatics21, 1-21 (2020).

[23] Miraskarshahi, R., Zabeti, H., Stephen, T. \& Chindelevitch, L. MCS2: minimal coordinated supports for fast enumeration of minimal cut sets in metabolic networks. Bioinformatics35, i615-i623 (2019).

[24] Pratapa, A., Balachandran, S. \& Raman, K. Fast-SL: an efficient algorithm to identify synthetic lethal sets in metabolic networks. Bioinformatics31, 3299-3305 (2015).

[25] Zhang, C., Ji, B., Mardinoglu, A., Nielsen, J. \& Hua, Q. Logical transformation of genome-scale metabolic models for gene level applications and analysis. Bioinformatics31, 2324-2331 (2015). 
[26] Tarjan, R. Depth-first search and linear graph algorithms. SIAM journal on computing1, 146-160 (1972).

[27] Herlihy, M. \& Shavit, N. The Art of Multiprocessor Programming, revised first edition. (Morgan Kaufmann, 2012).

[28] Orth, J. D., Thiele, I. \& Palsson, B. Ø. What is flux balance analysis? Nature biotechnology28, 245 (2010).

[29] Gudmundsson, S. \& Thiele, I. Computationally efficient flux variability analysis. BMC bioinformatics11, 1-3 (2010).

[30] Bundy, A. \& Wallen, L. in Catalogue of artificial intelligence tools 13-13 (Springer, 1984).

[31] Vempaty, N. R., Kumar, V. \& Korf, R. E. in AAAI. 434-440.

[32] Apaolaza, I., Valcarcel, L. V. \& Planes, F. J. gMCS: fast computation of genetic minimal cut sets in large networks. Bioinformatics35, 535-537 (2019).

[33] Jamshidi, N. \& Palsson, B. Ø. Investigating the metabolic capabilities of Mycobacterium tuberculosis $\mathrm{H} 37 \mathrm{Rv}$ using the in silico strain iNJ661 and proposing alternative drug targets. BMC systems biology1, 26 (2007).

[34] Hädicke, O. \& Klamt, S. EColiCore2: a reference network model of the central metabolism of Escherichia coli and relationships to its genome-scale parent model. Scientific reports7, 39647 (2017).

[35] Orth, J. D. et al. A comprehensive genome-scale reconstruction of Escherichia coli metabolism-2011. Molecular systems biology7, 535 (2011).

[36] Liao, Y.-C. et al. An experimentally validated genome-scale metabolic reconstruction of Klebsiella pneumoniae MGH 78578, i YL1228. Journal of bacteriology193, 1710-1717 (2011).

\section{Tables}

Table 1. Comparison of the number of LPs solved by Rapid-SL vs. Fast-SL for three GEMMs. 


\begin{tabular}{|llll|}
\hline & $\begin{array}{l}\text { Escherichia } \\
\text { coli }\end{array}$ & $\begin{array}{l}\text { Salmonella } \\
\text { Typhimurium }\end{array}$ & $\begin{array}{l}\text { Mycobacterium } \\
\text { tuberculosis }\end{array}$ \\
\hline Model name & iAF1260 [18] & STM_v1.0 [11] & iNJ661 [33] \\
\hline Medium & iM9/glucose & iM9/glucose & Middlebrook 7H9 \\
\hline Number of reactions & 2,382 & 2,546 & 1,028 \\
\hline $\begin{array}{l}\text { Number of exchange and diffusion } \\
\text { reactions* }\end{array}$ & 331 & 378 & 86 \\
\hline Number of reactions in $J_{\mathrm{nz}}$ & 406 & 484 & 414 \\
\hline Single lethal reactions & 278 & 329 & 309 \\
\hline Lethal reaction pairs & 96 & 152 & 75 \\
\hline Lethal reaction triplets & 247 & 275 & 140 \\
\hline Lethal reaction quadruplets & 402 & 1008 & 463 \\
\hline Total number of LPs solved: & & & $1.1910^{7}$ \\
\hline Fast-SL & $1.4510^{7}$ & $3.0110^{7}$ & $4.9010^{6}$ \\
\hline Rapid-SL & $7.3510^{6}$ & $1.9810^{7}$ & \\
\hline
\end{tabular}

* The exchange and diffusion reactions are not generally considered in the lethality analysis.

Table 2. The number of SLs and corresponding LPs solved in each group of branches of the Rapid-SL, while searching for single lethals to quadruple SLs in iAF1260. The branches are grouped based on the number of members in their starting node. Evaluating only the first group of branches identifies over $34 \%$ of SLs, while only about $0.65 \%$ of LPs must be examined.

\begin{tabular}{llll} 
Group identifier & Cardinality of the starting node & Number of SLs identified & $\begin{array}{c}\text { Number of } \\
\text { LPs solved }\end{array}$ \\
\hline I & One reaction & 254 & 47,869 \\
\hline II & Two reactions & 220 & 646,501 \\
\hline III & Three reactions & 192 & $2,679,417$ \\
\hline IV & Four reactions & 79 & $3,974,889$
\end{tabular}

Table 3. SLs identified by evaluation of only the branches with one reaction in the starting node (Group I). 


\begin{tabular}{|c|c|c|c|c|}
\hline Model & Cardinality of SLs & All SLs & Enumerated in Group (I) & Fraction (\%) \\
\hline \multirow{5}{*}{ iJ01366 } & Double SL & 259 & 237 & 91.5 \\
\hline & Triple SL & 1,162 & 871 & 75.0 \\
\hline & Quadruple SL & 3,585 & 2,275 & 63.5 \\
\hline & Total SLs & 5,006 & 3,383 & 67.6 \\
\hline & Total number of LPs solved & $32,615,092$ & 322,670 & 1.0 \\
\hline \multirow{5}{*}{ iYL1228 } & Double SL & 146 & 127 & 87.0 \\
\hline & Triple SL & 289 & 178 & 61.6 \\
\hline & Quadruple SL & 1090 & 513 & 47.1 \\
\hline & Total SLs & 1,525 & 818 & 53.6 \\
\hline & Total number of LPs solved & $12,283,617$ & 125,159 & 1.0 \\
\hline
\end{tabular}

Table 4. Results of three introduced applications.

Search through a Applying constraints on the specific list of targets

\begin{tabular}{ll}
\hline Model Nam \\
\hline Medium \\
\hline Double SLs \\
\hline Triple SLs \\
\hline Quadruple \\
\hline Quintuple S \\
\hline Sextuple S \\
\hline Septuple S \\
\hline Octuple SL \\
\hline Total numb \\
of LPs solved \\
Figures
\end{tabular}
branching of the DFS

Selective enumeration among the DFS branches

iAF1260

iM9/glucose

74

98

41

82

11

159

59

307

125

476

402

9126

$27,803,258$

$89,958,961$ 


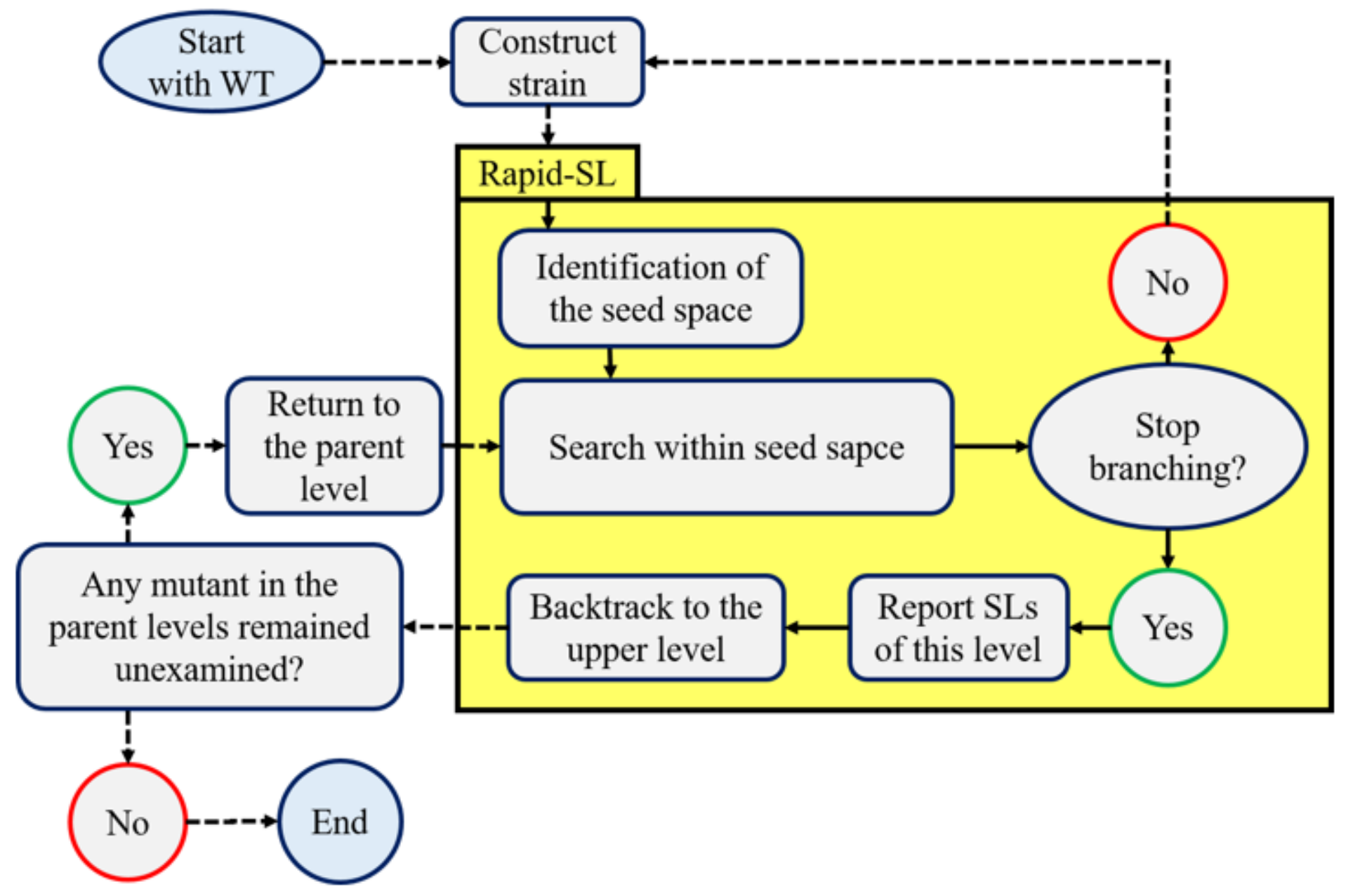

Figure 1

The flowchart of Rapid-SL. Calling Rapid-SL from itself represents the recursive feature of our implementation. 

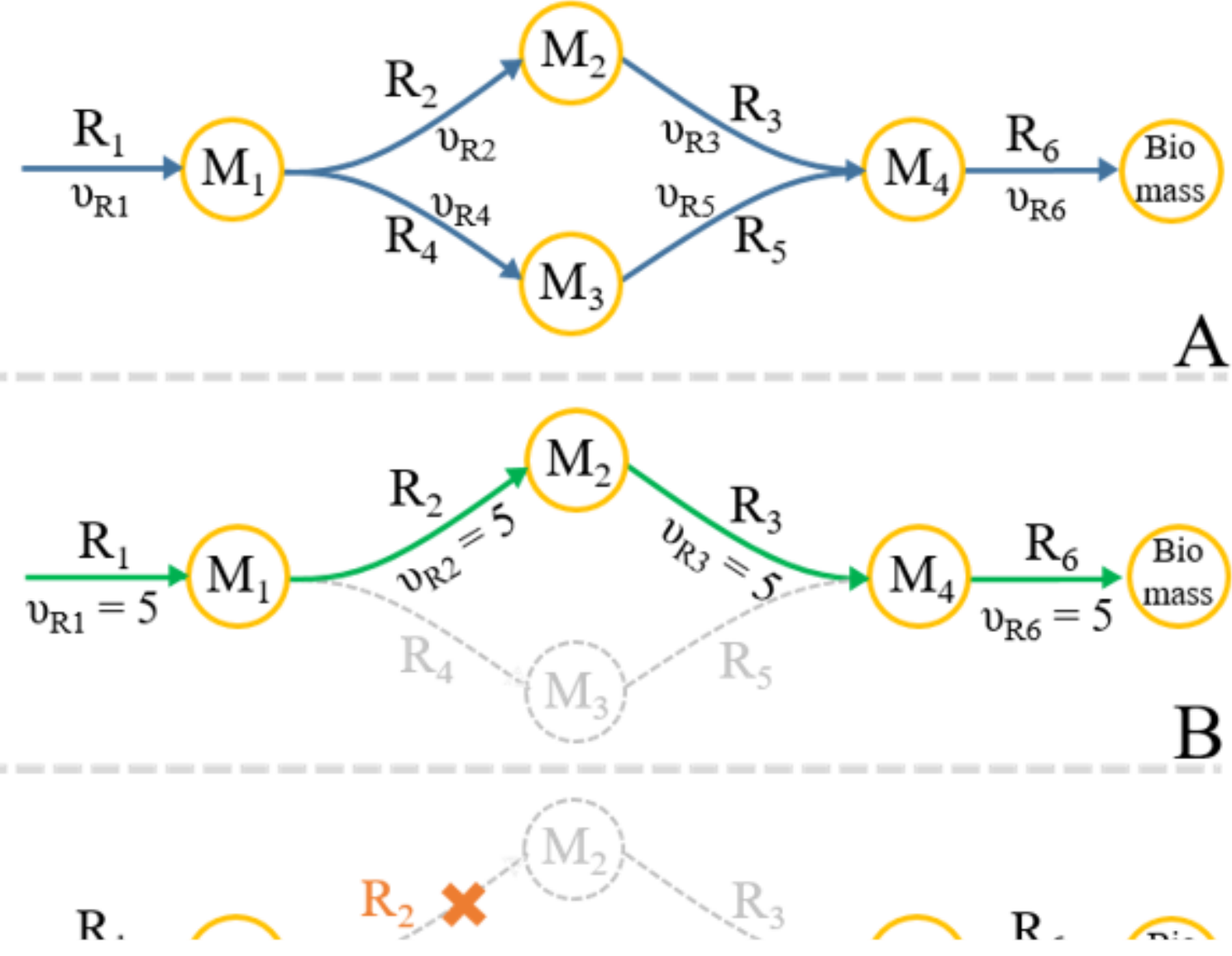

Figure 2

The effect of removing a non-lethal reaction of $J_{n z}$. (A) Toy model; $R_{i}$ denotes the reaction names, and $U_{R i}$ represents the flux through $R_{i}$. (B) Flux distribution of the wild-type strain. $J_{n z}=\left(R_{1}, R_{2}, R_{3}, R_{6}\right)$. (C) Flux distribution of the mutant strain in which $R_{2}$ is removed. In order to maximize the flux of $R_{6}$, the $R_{4}$ and $R_{5}$ reactions gain nonzero fluxes unlike the wild-type strain. Therefore, removing any of the activated reactions $\mathrm{R}_{4}$ or $\mathrm{R}_{5}$ will block the flux through $\mathrm{R}_{6}$ and the biomass objective function. 


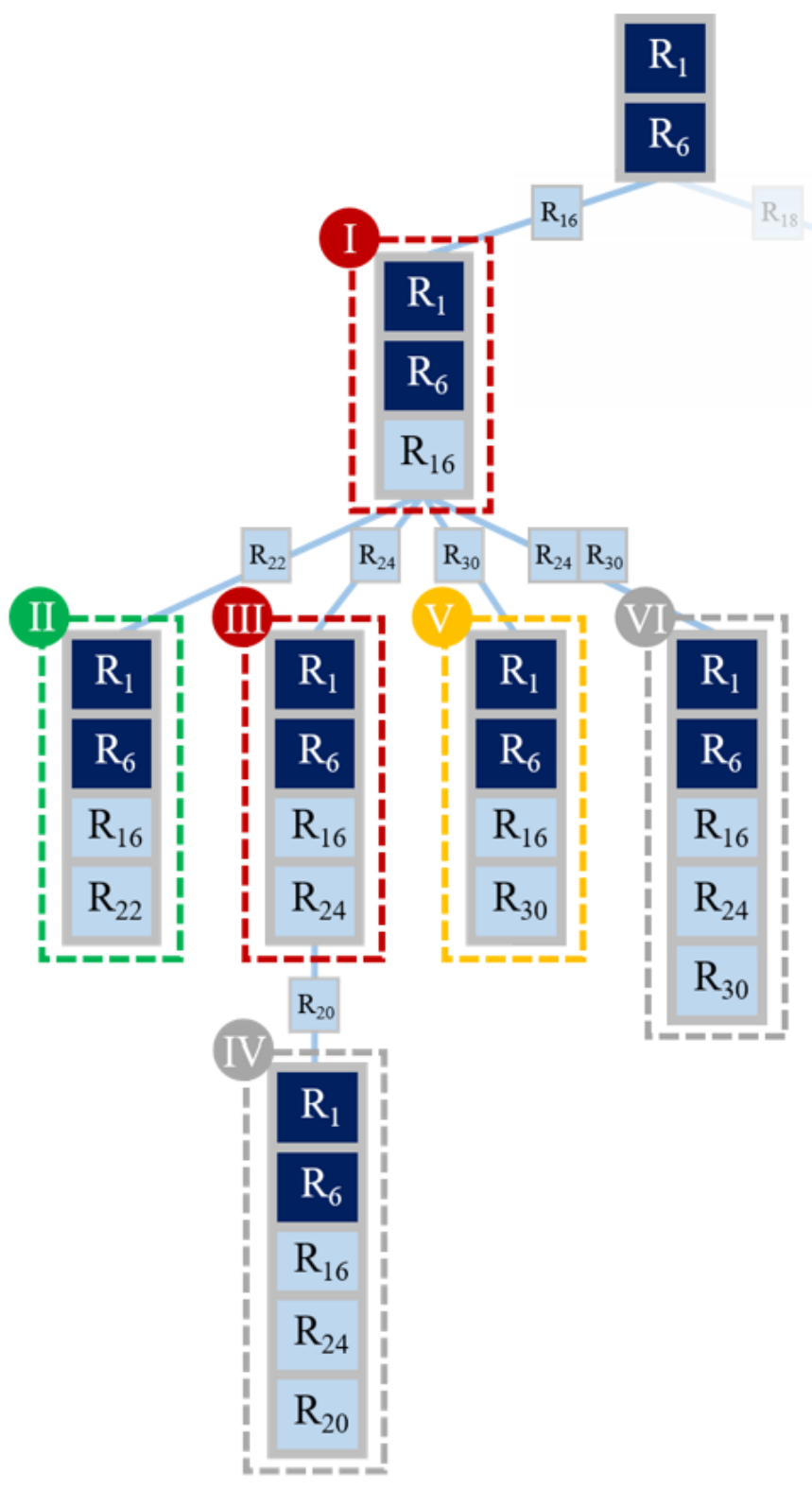

Figure 3. Schematic of a typical example of the depth-first search in our implementation. The squares represent targets. Here $\left(\mathrm{R}_{1}, \mathrm{R}_{6}\right)$ is a non-lethal set from the second step. The Roman numerals show the order of progress in examining the lethality of different sets. Light blue squares represent the targets that gain non-zero fluxes after removing some reactions. Node I as well as node III are non-lethal sets and their removal activates new potential targets (๑). Node II shows a lethal set and thus branching from this node is stopped (๑). Branching in node $\mathrm{V}$ is stopped because no new potential target could be activated (O). Branching in nodes IV and VI are stopped because the maximum desired cardinality is reached $(\odot)$. After examining the sets corresponding to the $\mathrm{R}_{16}$ branch, the process will continue for the $\mathrm{R}_{18}$ branch.

\section{Figure 3}

See image above for figure legend.

\section{Supplementary Files}

This is a list of supplementary files associated with this preprint. Click to download. 
- Supplementaryfiles.zip

Page 18/18 